\title{
POTENCIAL PRODUCTIVO DE LA RAZA BOVINA SERRANA DE TERUEL. RESULTADOS PRELIMINARES
}

\author{
PRODUCTIVE POTENTIALOF THE BOVINE SERRANA DE TERUEL BREED. \\ PRELIMINARY REPORT
}

\author{
Sanz, A. ${ }^{1 *}$, Ripoll, G. ${ }^{1}$, Blasco, I. ${ }^{1}$, Álvarez-Rodríguez, J. ${ }^{1}$ y Albertí, P. ${ }^{1}$ \\ ${ }^{1}$ Centro de Investigación y Tecnología Agroalimentaria de Aragón (CITA). Avda. Montañana 930. 50059 \\ Zaragoza. España. *asanz@aragon.es
}

\section{Palabras clave adicionales}

Razas amenazadas. Canal. Carne.

\section{RESUMEN}

En el marco de un proyecto de recuperación de la raza Serrana de Teruel, se estudió la calidad de la canal de las categorías comerciales de ternero, añojo y cebón (los últimos castrados con 9 meses), con edades a sacrificio de 12,22 y 22 meses, y pesos vivos de 470, 720 y $660 \mathrm{~kg}$, respectivamente. Veinte terneros se sometieron a cebo convencional con paja y concentrado a voluntad, desde los 4 meses hasta el sacrificio, excepto entre los 15 y 20 meses, en que añojos y cebones recibieron ensilado de cebada a voluntad y $3 \mathrm{~kg}$ concentrado/animal/día. El primer año de vida, la ganancia de peso de los terneros fue de $1,5 \mathrm{~kg} /$ día; el segundo año, fue superior en añojos que en cebones ( $1,1 \mathrm{vs}$. 0,9 kg/día), como consecuencia de la castración. Las canales de los animales enteros presentaron mayor rendimiento y conformación, y menor engrasamiento que las de los cebones ya que la castración favoreció la deposición de grasa intramuscular. Según los resultados, la raza se encuadró entre las rústicas bovinas españolas. Se abren alternativas de producción como el cebón, susceptibles de acogerse a distintivos de calidad diferenciada, que deberían suponer un incentivo para la explotación de la Serrana de Teruel frente a otras razas, lo que garantizaría su conservación a largo plazo.

\section{SUMMARY}

The carcass quality from three commercial categories (yearling, bull and steer castrated at 9

Presentado al Congreso SERGA (2010, Asturias).

Recibido: 3-12-10. Aceptado: 13-4-11.

\author{
Additional KEYWORDS \\ Endangered breeds. Carcass. Beef.
}

months old) was studied within the framework of a recovery programme in the Serrana de Teruel breed. Ages at slaughter were 12, 22 and 22 months, and live-weights were 470, 720 and 660 $\mathrm{kg}$, in yearling, bull and steer, respectively. Twenty calves were managed under a conventional feeding programme of ad libitum concentrate and straw from 4 months old to slaughter, except between 15 and 20 months old, in which bulls and steers received ad libitum barley silage as forage supplemented with $3 \mathrm{~kg}$ of concentrate/head/day. The gain during the first year of age was $1.5 \mathrm{~kg} /$ day, whereas during the second year it was greater in bulls than in steers $(1.1 \mathrm{vs} .0 .9 \mathrm{~kg} /$ day $)$, as a consequence of castration. Bull carcasses had greater dressing percentages and conformation scores and lower fatness degree than steers, confirming a castration-induced intramuscular fat deposition. Considering these results, Serrana de Teruel breed falls within the group of local Spanish bovine breeds. These studies should provide the conditions for a labelled beef product that allows the farmer's survival and the assurance of Serrana de Teruel breed conservation for a long term.

\section{INTRODUCCIÓN}

La raza Serrana de Teruel proviene del Bos taurus primigenius, posteriormente enlazado con el Bos taurus ibericus y constituye una variante de las razas bovinas que se desarrollaron en las serranías del Sistema Central (Aparicio, 1944). Tanto por su ubica- 
SANZ, RIPOLL, BLASCO, ÁLVAREZ-RODRÍGUEZY ALBERTí

Tabla I. Pesos, clasificación y medidas morfométricas de las canales de los 3 tipos comerciales. (Weights, classification and carcass morphometric measures of the 3 commercial categories).

\begin{tabular}{|c|c|c|c|c|c|}
\hline & Ternero & Añojo & Cebón & EE & Sig \\
\hline Peso vivo sacrificio $(\mathrm{kg})$ & $471,3^{c}$ & $720,3^{a}$ & $660,6^{b}$ & 18,7 & $* \star *$ \\
\hline Peso canal fría $(\mathrm{kg})$ & $277,4^{c}$ & $425,7^{a}$ & $365,2^{b}$ & 10,1 & $* * *$ \\
\hline Rendimiento canal (\%) & $58,9^{a}$ & $59,1^{\mathrm{a}}$ & $55,3^{\mathrm{b}}$ & 0,4 & *** \\
\hline Conformación (1-18) & $10,3^{a}$ & $9,7^{a}$ & $8,3^{b}$ & 0,4 & $* *$ \\
\hline Engrasamiento (1-15) & $5,0^{\mathrm{b}}$ & $5,7^{\mathrm{b}}$ & $8,0^{\mathrm{a}}$ & 0,3 & $* \star *$ \\
\hline Longitud canal (cm) & $121,7^{b}$ & $140,6^{a}$ & $137,7^{a}$ & 1,2 & $* \star *$ \\
\hline Anchura canal (cm) & $58,6^{b}$ & $68,2^{\mathrm{a}}$ & $67,4^{a}$ & 0,6 & *** \\
\hline Profundidad interna pecho $(\mathrm{cm})$ & $32,7^{b}$ & $38,6^{a}$ & $39,3^{a}$ & 0,9 & *** \\
\hline Longitud pierna $(\mathrm{cm})$ & $78,6^{b}$ & $89,4^{\mathrm{a}}$ & $88,0^{a}$ & 0,9 & $* \star *$ \\
\hline Anchura pierna (cm) & $27,3^{c}$ & $31,5^{\mathrm{a}}$ & $29,8^{b}$ & 0,4 & $* * *$ \\
\hline Perímetro pierna (cm) & $114,8^{c}$ & $129,8^{a}$ & $122,8^{b}$ & 1,2 & $* * *$ \\
\hline Profundidad pierna $(\mathrm{cm})$ & $42,9^{b}$ & $47,7^{\mathrm{a}}$ & $46,0^{\mathrm{a}}$ & 0,6 & $* * *$ \\
\hline
\end{tabular}

${ }^{a b}$ En cada línea, distinta letra entre tipos comerciales indica diferencias significativas $(p<0,05)$.

ción como por su sistema de explotación, es representativa de la primitiva agrupación Serrana. Se trata de una población muy rústica y adaptada a las duras condiciones de montaña seca, utilizada tanto para la

Tabla II. Composición tisular y despiece comercial de las canales de los 3 tipos comerciales estudiados. (Tissue composition and beef cuts of the 3 commercial categories).

\begin{tabular}{|c|c|c|c|c|c|}
\hline & Ternero & Añojo & Cebón & $n$ EE & \\
\hline Carne (\%) & $74,4^{a}$ & $75,8^{\mathrm{a}}$ & $71,4^{b}$ & 0,7 & ** \\
\hline Grasa ( & & $5,1^{\mathrm{b}}$ & $8,4^{a}$ & 0,4 & *** \\
\hline Hueso (\%) & 20,1 & 19,1 & 20,3 & 0,5 & \\
\hline Extra (\%, solomillo) & $3,1^{a}$ & $2,8^{b}$ & $3,1^{\mathrm{a}}$ & 0,07 & \\
\hline Primera $^{1}(\%)$ & $63,4^{a}$ & $61,4^{\mathrm{b}}$ & $60,9^{b}$ & 0,4 & $*$ \\
\hline Segunda $^{2}(\%)$ & 7,2 & 7,1 & 7,3 & 0,1 & ns \\
\hline Tercera $^{3}(\%)$ & $26,4^{b}$ & $28,8^{a}$ & $28,8^{a}$ & 0,5 & ** \\
\hline
\end{tabular}

abEn cada línea, distinta letra entre tipos comerciales indica diferencias significativas $(p<0,05)$.

${ }^{*} p<0,05 ;{ }^{* *} p<0,01 ;{ }^{* * *} p<0,001$; ns: no significativo. ${ }^{1}$ Lomo+babilla+tapa+contra+culata contra+cadera+rabillo+redondo+aguja+espalda+pez.

${ }^{2}$ Morcillos+llana+aleta+brazuelo.

${ }^{3}$ Falda+diafragma+filete de rellenar+pecho+pescuezo+costillar+trapillos+recortes. producción de carne y piel como para el trabajo rural. En la segunda mitad de siglo $\mathrm{XX}$, la raza fue cruzada con otras más selectas, presentando una evolución censal claramente regresiva.

En el año 2000 se iniciaron las labores de caracterización y conservación con 60 individuos (Vijil et al., 2009), y en la actualidad el censo ha ascendido a 240 individuos. El interés en su conservación se tradujo en la constitución de la Asociación de ganaderos (ASERNA) y la creación de los núcleos de conservación in situ (Diputación Provincial de Teruel, Cedrillas (Teruel)) y conservación ex situ (CTA del Gobierno de Aragón, Movera (Zaragoza)). Se resumen a continuación algunos de los resultados obtenidos en la evaluación del potencial productivo de la raza Serrana de Teruel, como base para la obtención de productos cárnicos susceptibles de acogerse a distintivos de calidad diferenciada.

\section{MATERIAL Y MÉTODOS}

Se utilizaron 20 terneros machos de raza Serrana de Teruel con un peso al destete del $148 \mathrm{~kg}$ (peso vivo nacimiento $38 \mathrm{~kg}$; edad destete 141 días; ganancia nacimiento-des-

Archivos de zootecnia vol. 60, núm. 231, p. 378. 
tete: $0,748 \mathrm{~kg} / \mathrm{d}$ ). Los terneros se sometieron a un cebo convencional con pienso y paja a voluntad desde su destete hasta alcanzar la edad objetivo al sacrificio, excepto entre los 15 y 20 meses de edad, en que añojos y cebones recibieron ensilado de cebada a voluntad y un suplemento de $3 \mathrm{~kg}$ concentrado/animal/día. Las categorías comerciales evaluadas fueron ternero, añojo y cebón (según el R.D. 75/2009), con edades a sacrificio de 12, 22 y 22 meses, y pesos vivos de 470,720 y $660 \mathrm{~kg}$, respectivamente. La castración en los terneros destinados a la producción de cebón se realizó con 9 meses de edad. Durante el período de engorde, se registró el peso semanal de los terneros.

Después del sacrificio de los animales, se registró el peso de las canales calientes y se aplicó una pérdida del $2 \%$ para estimar el peso de la canal oreada. Tras 24 horas de oreo a $4^{\circ} \mathrm{C}$, las canales fueron clasificadas según el Reglamento CEE 1026/91, realizando la valoración de la conformación (escala 1-18) y el engrasamiento (escala 1-15). Se realizó el despiece de la media canal izquier- da, determinando la composición tisular en porcentaje sobre la canal de carne comercializable, grasa de recorte y hueso, y la composición comercial por el porcentaje de carne de las categorías extra, $1^{\mathrm{a}}, 2^{\mathrm{a}}$ y $3^{\mathrm{a}}$. Se tomaron muestras del músculo Longissimus dorsi ( $5^{\mathrm{a}}$ a $11^{\mathrm{a}}$ costilla) para realizar el análisis instrumental de la carne. Se midió el pH en el músculo a la altura de la $10^{\mathrm{a}}$ costilla y la dureza instrumental del músculo a tres tiempos de maduración (1, 7 y 14 días) en un aparato Instron con una sonda WarnerBratzler. Las diferencias entre categorías se estudiaron mediante análisis de varianza.

\section{RESULTADOSYDISCUSIÓN}

En el primer año de vida, la ganancia de peso de los terneros fue de $1,5 \mathrm{~kg} /$ día; durante el segundo año, la ganancia fue superior en añojos que en cebones $(1,1$ vs. $0,9 \mathrm{~kg} /$ día), como consecuencia de la castración. Las canales de los animales enteros presentaron mayores valores en rendimiento y conformación, e inferior grado de engrasa-

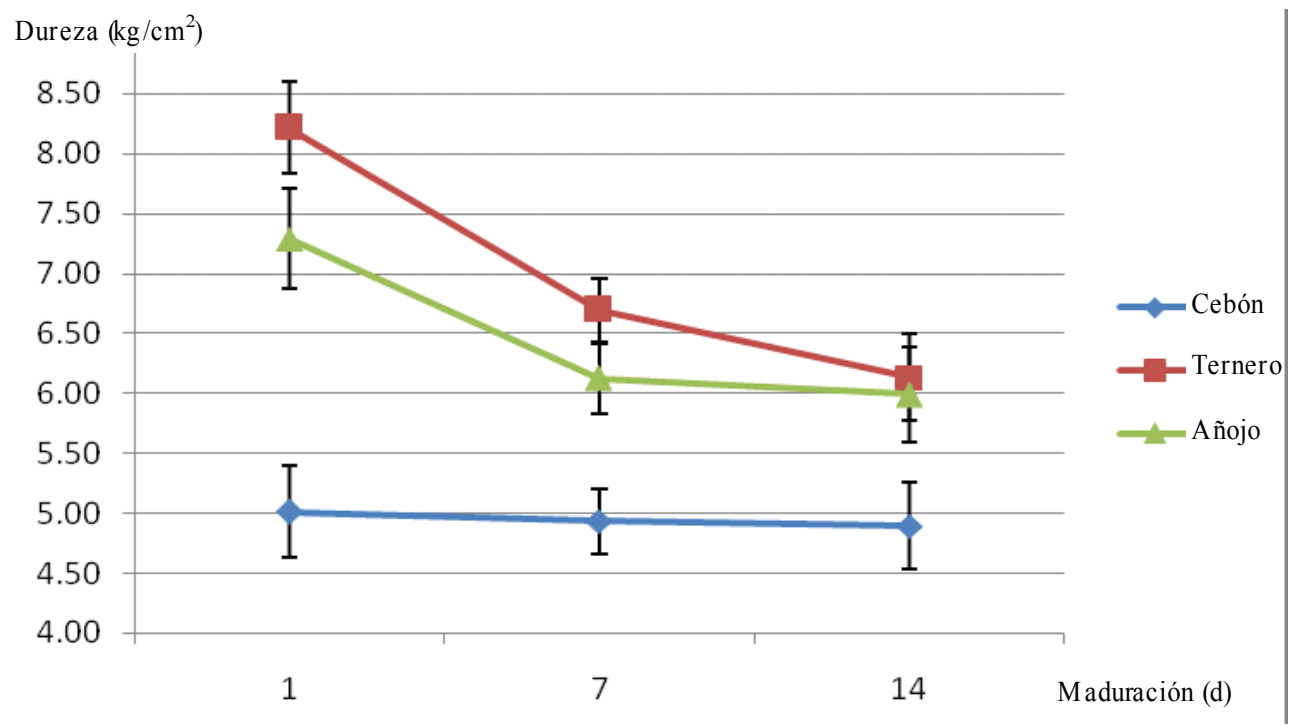

Figura 1. Evolución de la textura instrumental (esfuerzo máximo). (Evolution of the instrumental texture (maximum load)). 
miento que las procedentes del lote de cebones (tabla I), confirmando que la castración favoreció la deposición de grasa intramuscular. Obviamente, las medidas objetivas de conformación de la canal se incrementaron con la edad de los animales. La castración afectó de forma significativa a la anchura y perímetro de la pierna, presentando los cebones inferiores valores a los animales añojos.

El pH de la carne fue normal en las tres categorías (media de 6), correspondiente a animales que no han sufrido estrés previo al sacrificio, no presentando, a priori, efectos negativos sobre la calidad de la carne. Como puede observarse en la tabla II, las categorías ternero y añojo ofrecieron un porcentaje superior de carne respecto a la categoría cebón, que a su vez mostró el porcentaje más elevado de grasa. De forma global en los tres tipos comerciales estudiados en la raza Serrana de Teruel, las canales presentaron un elevado porcentaje de hueso, superior al registrado en otras razas rústicas, una proporción de grasa baja y un porcentaje de carne alto, intermedio a los valores obtenidos en el estudio de Albertí et al. (2001), para las razas rústicas y razas cárnicas españolas. Por otro lado, la categoría añojo presentó inferior porcentaje de carne extra al observado en las categorías ternero y cebón.

La dureza instrumental de las carnes de ternero y añojo disminuyó con el tiempo de

\section{BIBLIOGRAFÍA}

Albertí, P., Sañudo, C., Olleta, J.L., Panea, B. y Lahoz, F. 2001. Efecto del peso de sacrificio en el rendimiento cárnico de terneros de siete razas bovinas españolas. ITEA, (Extra 22): 511 513.

Asenjo, B. 1999. Efecto de la raza y de la alimentación en los parámetros productivos y de calidad de canal y de carne en añojos de razas Charolés y Serrana Soriana. Tesis Doctoral. Universidad de Valladolid. 225 pp.

Aparicio, G. 1944. Zootecnia especial. Etnología compendiada. Descripción y estudio de razas maduración (figura 1), aunque se observó mayor terneza en la carne de añojo a 1 y 7 días de maduración que en la de ternero, desapareciendo esa diferencia a los 14 días. La dureza obtenida en la carne de añojo a los 7 días de maduración fue ligeramente inferior a la registrada por Asenjo (1999) en animales de raza Serrana Negra $\left(7,2 y 7,7 \mathrm{~kg} / \mathrm{cm}^{2}\right.$, para pienso comercial y natural) y superior a la observada en otras razas españolas (Sañudo et al., 2004). Finalmente, la dureza instrumental registrada en la carne de la categoría cebón fue muy inferior a la del resto de categorías, y en los tres tiempos estudiados, debido probablemente a la mayor proporción de grasa intramuscular observada en este lote de animales.

En conclusion, con relación a la precocidad, conformación, nivel de engrasamiento y características de calidad de carne, la raza se encuadra entre las rústicas bovinas españolas. Se abren alternativas de producción como el cebón, susceptibles de acogerse a distintivos de calidad diferenciada, que deberían suponer un incentivo para la explotación de la Serrana de Teruel frente a otras razas, lo que garantizaría su conservación a largo plazo.

\section{AGRADECIMIENTOS}

Trabajo financiado por el proyecto INIA PET2007-05-C03-01 y fondos FEDER.

nacionales y extranjeras. Imprenta Moderna. Córdoba. España. 242 pp.

Sañudo, C., Macie, E.S., Olleta, J.L, Villarroel, M., Panea, B. and Albertí, P. 2004. The effects of slaughter weight, breed type and ageing time on beef meat quality using two different texture devices. Meat Sci., 66: 925-932.

Vijil, E., Picot, A., Hernández, M., Pastor, F., Quintín, F.J., Sevilla, E., Abril, F. y Sanz, A. 2009. Raza bovina Serrana de Teruel: estructura poblacional, características reproductivas y maternales. Arch. Zootec., 58 (Supl. 1): 509-512. 